This material is published in the open archive of Mid Sweden University

DIVA http://miun.diva-portal.org

to ensure timely dissemination of scholarly and technical work. Copyright and all rights therein are retained by authors or by other copyright holders. All persons copying this information are expected to adhere to the terms and constraints invoked by each author's copyright. In most cases, these works may not be reposted without the explicit permission of the copyright holder.

Sjöström, M.; Olsson, R.; Dalin, R., "A digital 3D signage system and its effect on customer behavior," The International Conference on 3D Imaging (IC3D) 7-8 Dec. 2011, 2011.

(C) 2011 IEEE. Personal use of this material is permitted. However, permission to reprint/republish this material for advertising or promotional purposes or for creating new collective works for resale or redistribution to servers or lists, or to reuse any copyrighted component of this work in other works must be obtained from the IEEE by sending an email to pubs-permissions@ieee.org. 


\title{
A DIGITAL 3D SIGNAGE SYSTEM AND ITS EFFECT ON CUSTOMER BEHAVIOR
}

\author{
Mårten Sjöström, Roger Olsson, and Rolf Dalin
}

\author{
Mid Sweden University, SE-85170 Sundsvall, Sweden
}

\begin{abstract}
The use of digital signs simplifies distribution. Importantly, it draws more attention than static signs. A way to increase attention is to add an experienced depth. The paper discusses possible alternatives for extending an existing digital signage system to display stereoscopic $3 \mathrm{D}$ contents, comparing a decentralized distribution solution and a centralized solution. A functional prototype system was implemented. A new 3D player was developed to render views from different formats. The implemented system was used to study customer behavior when exposed to digital stereoscopic $3 \mathrm{D}$ signage in a direct sales situation. The proportion of sales of selected products related to the total number of sold products varied approximately equally before and during tests. An interview study suggests that the sign did not interact with customer decisions: customers were lost at different stages in this series of steps, among others the sign placement.
\end{abstract}

Index Terms - 3D displays, signage, system integration, stereoscopic 3D content, distribution, customer evaluation.

\section{INTRODUCTION}

In recent years, research advances in stereoscopic threedimensional (3D) visualization leaves room for fundamentally changing the way in which all 3D information is presented. The 3D technology may augment experience in several areas where current presentation methods primarily consist of ordinary two-dimensional displays without depth, e.g. medical diagnostics, product design and engineering, digital billboards, video games and 3DTV, mobile etc. The question arises what the required adjustments to an existing system are in order to present stereoscopic 3D, and what the benefits it brings.

So-called auto-stereoscopic 3D displays have left the prototype stage and make it possible to create a lifelike 3D effect where the viewer is experiencing a real-world depth of the image without having to wear special glasses [1]. A number of different perspectives of a scene are projected out into the room by which a viewer experiences both depth and can look around objects in the presented scene [2]. When objects are literally coming out of the display, the ability to attract the attention of the passers-by may increase and turn him or her into a customer.

Digital signage is a growing part of overall advertising offering. The explosion of recent years - largely due to the

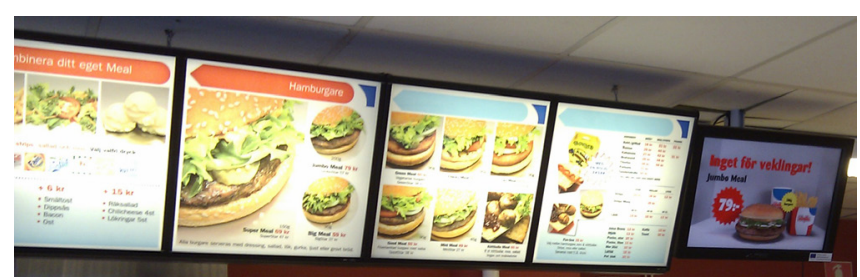

Fig. 1. Restaurant menus and a digital 3D sign

technical success of flat panel displays such as LCD - will make the digital advertising turn into a multi-billion U.S. dollar industry [3]-[4]. Analysts indicate that the rapid growth in the area will continue in the 2010 decade [5]. Despite the similarity with other marketing channels such as television and cinema, knowledge and experience in advertising production for these media are only partially exploited. The content presented on the digital signs must capture the viewer's interest over a much shorter time, when attention and patience are smaller in the shop than in your living room or in the cinema. One way to increase the customers' attention is to add additional features to the digital sign. A following step would be to present the material with lifelike depth.

3D signage (see Fig. 1) is a self sustained system in the sense that the whole chain from production to presentation is under the control of the system owner. It does not interact with other systems and can therefore adopt special and propriety solutions. Standardization for 3D signage will, nonetheless, allow for cross-platform solutions. Predicted advantages with 3D signage include a sensational effect that can be exploited to draw the attention of customers; the reported risk of nausea, headaches etc. [6] would be very limited as viewers are exposed for a very limited time; and the public awareness of 3D media and cinema increases the attention of signs with the same effect. A drawback with 3D signage is of course the increased costs of the technology, and also the fact that $5-10 \%$ of the population cannot experience depth through binocular parallax [7]. However, multi-view displays allow these persons to experience depth by moving their position slightly, i.e. motion parallax.

The current paper presents an investigation of possible alternatives for extending an existing digital signage system to allow for 3D media to be presented on 3D displays. The digital signage distribution chain has been analyzed considering 3D content production, presentation, storage and distribution. In particular, a comparison of a centralized and a de-centralized distribution model has been made. Based on the analysis of the distribution chain, a prototype 
digital 3D signage system has been implemented. It comprises, among others, a newly developed, stand-alone, modular, GPU-based, cross-platform 3D video player, which makes the solution adjustable to different compression methods and various 3D displays. The prototype system was utilized to study customer behavior when exposed to digital 3D signage in a direct sales situation. In a quantitative investigation, sales were compared to the displayed contents. A supplementary qualitative investigation was carried out based on the market ladder model.

Outline of the paper is as follows. Section 2 presents an overview of the signage distribution chain and the options for a digital 3D signage system. Section 3 describes the existing signage system and the choices made for its extension to a 3D signage system. In Section 4, we present our study on customer behavior as they are exposed to a digital 3D signage system. A conclusion is given in Section 5.

\section{SIGNAGE DISBRIBUTION CHAIN}

This section gives an overview of the distribution chain from content production to customer presentations, options and aspects on the different parts of the distribution chain.

\subsection{Content Production}

One of the more important issues with digital 3D signage is the production of $3 \mathrm{D}$ contents. It is desired to allow both for computer generated (CG) contents, real life photographic media, and a mixture of these.

Photorealistic 3D scenes can be produced in commercial modeling, animation and rendering software such as Autodesk's 3ds Max or Maya, or others. An advantage with this CG-based 3D content is that it easily facilitates changing scene properties such as object staging, lighting and animation. The rendering step is often extended by a plug-in software, which converts the $3 \mathrm{D}$ content to a format optimized for a specific display or display type. Despite the simplicity of this approach, such display specific formats cannot be utilized when a more general distribution of $3 \mathrm{D}$ content is required. This is mainly because storing multiple views in an interlaced manner is convenient for direct presentation on a lenticular or parallax barrier display, but it is very inefficient when applying a compression scheme to it. In the near future 3D content will have to be display agnostic, much in the same way that $2 \mathrm{D}$ content has been for a long time. This can be done by rendering to a generic $3 \mathrm{D}$ format that allows the content to be adjusted on the client side such that it fits the properties of that specific 3D display.

Photographic production of $3 \mathrm{D}$ content implies a great increase in work and costs. Already for 2D film production, the shooting with crew, actors and equipment requires substantial financing. For 3D content production, both additional equipment such as stereo cameras or multi- camera rigs, as well as crew such as stereographer result in an amplified cost. Even though photographic 3D production has a similar number of processing steps as for CG based $3 \mathrm{D}$, the complexity of producing high quality multi view $3 \mathrm{D}$ content increases when virtual scenes and cameras are replaced with real life equivalents. Professional stereoscopic 3D film production in both Hollywood and Europe has gathered important experience for producing high quality results [8]. When more than two views are required, a multicamera rig may be adopted. The same amount of cameras may be used as there are views on the $3 \mathrm{D}$ display that is intended for presentation. This reduces post-processing, but limits the flexibility to use the contents between different kinds of 3D displays. A more flexible capture approach is to produce depth-maps approximating the 3D scene from stereo-pairs, and possibly supplemented with range cameras for a better depth-map creation [9]. Independent of number of views, post-production is required, which includes compensation between cameras, such as color correction, right-left image rectification and vertical shift, but also adjustment of disparity (horizontal shift) in order to achieve the desired depth experience.

Applying dynamic advertising text to the $3 \mathrm{D}$ content requires special care unless it is a part of the $C G$ or photographic 3D scene. Text should be applied in such a way that fixational eye movement and conflict between depth cues (mainly binocular parallax, occlusion and window violation) are avoided. In practice, this means that text should be slightly in front of the region of interest.

\subsection{Presentation}

A requirement for digital $3 \mathrm{D}$ signage is the use of autostereoscopic displays, i.e. without using special spectacles for separating left and right view. Today there is a variety of $3 \mathrm{D}$ displays [10]-[11]. The common ability is the projection of a certain number of views into the viewing space. Size, resolution (number of pixels), number of projected views and angle between views are all different and have been adjusted for an optimal viewing distance. Furthermore, view data must be rearranged into specific pixel configurations such that the views are projected correctly into the viewing space by the optics of the displays. This means that each type of 3D display has its own characteristic for which the views must have been produced.

The stereoscopic 3D (S3D) format, i.e. two perspective views of the scene, implies substantial processing on the client side in order to compute additional, intermediate, views to be displayed on autostereoscopic 3D displays. The extension of views in the multiview format means that there are as many (or more) perspective views as the $3 \mathrm{D}$ display may present. It overcomes deficiency of substantial processing but is less flexible for different 3D displays. The format $2 \mathrm{D}$ plus depth implies that the $2 \mathrm{D}$ video is extended with a depth value per pixel. It allows for flexibility in 
computing different number of views, but does not produce as good quality as multiview with a correct number of views. Quality may be improved over 2D plus depth by using the formats depth-enhanced stereo (DES), multiview plus depth (MVD), or layered depth video (LDV), for which there exist compression algorithms [12]. DES consists of $\mathrm{S} 3 \mathrm{D}$ video with depth information per pixel in each view. MVD is the extension of DES to contain more than two perspective views with corresponding depth information. LDV extends the 2D plus depth format with 2D and depth information of hidden surfaces such as background.

A signage solution should be highly flexible in the sense that it should support a multitude of formats and compression standards for multiview presentations on a variety of $2 \mathrm{D}$ and $3 \mathrm{D}$ displays running on different computer platforms.

\subsection{Storage and Distribution}

Finalized 3D contents must be easily handled by the existing digital signage system. This means that the format for storage, and protocols for distribution, must be selected appropriately. This includes decisions about what kind of encoding formats are suitable for the respective $3 \mathrm{D}$ contents.

Distribution is normally not time-critical for digital signage, whereas quality is essential. Therefore, a reliable distribution is preferably carried out by offline point-topoint error-free transmission. This implies a secured quality of the contents whereas varying channel conditions merely imply a delayed transmission.

\subsubsection{Decentralized solution}

A digital signage system should be able to present both images and motion pictures. Most media players allow to decoding and presenting contents in different formats and encoding. Such a decentralized distribution solution can ignore the actual contents, formats and encoding of a media file, and let display node discern the content structure and corresponding processing, e.g. view rendering from different storage formats. This simplifies the structure of a distribution system, but requires a more complex and complete media player at the display node. Such a complete media player at each display node permits the same contents to be displayed on different display types (2D and multiview of different number of views) reusing the same media file; the media player then adjusts the contents to the specific display, based on a flexible representation format such as 2D plus depth, MVD, DES and LDV. The sacrifice related to this flexibility is the requirement of a powerful graphical processor at the display site where 3D contents shall be presented on a multiview display. This solution implies that the media player shall be informed of the 3D display's size, number of views, etc. by a configuration file. The operator of the system may be alerted in case of an error or a faulty format is sent to the display node.



Fig. 2. Web-based interface for managing the contents distributed by the digital signage system

\subsubsection{Centralized solution}

An alternative centralized distribution system keeps track of allowed formats for each display node. In this way, appropriate formats are distributed to each separate node. This requires less powerful processors at display sites, but it necessitates different formats for the same contents, resulting either in larger central storage or a transcoding before transmitting contents, the latter implying a central powerful graphical processor in order to reduce delays in distribution. The processing power at sites with 3D presentation capability may also be diminish if contents already have been adapted to the specific 3D display before transmission. The files to transfer are then not in a format that easily is encoded, which make them enormously large. Furthermore, that solution requires a large and very fast memory for presenting the contents.

\section{INTEGRATION OF 3D CAPABILITY}

\subsection{Existing Digital Signage system}

The existing digital signage system is a complete system in the sense that it consists of hardware and software to present digital contents and managing it remotely. The software is completely web-based and is used directly by customers to update and modify content on the displays at both central and local levels, see Fig. 2. This means that the software is tailored to manage one or several displays or even parts of displays, so that different users can access these advertisement spots independently. The signage system automatically recognizes a large number of file formats for static or motion media, which are transparent to the management software. The software further allows adding and changing superimposed text in different ways. 


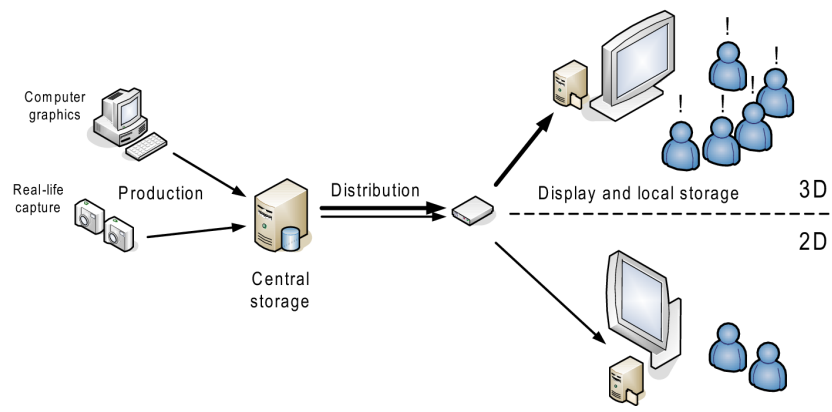

Fig. 3. Digital 3D signage system with 2D capabilities

The existing system constitutes a decentralized solution as the decoding and processing of existing media files are carried out at the display nodes. It has a centralized long term storage and temporary storage of media files in the nodes at the sites of media exposure. This client-server solution allows the client to play a large number of different media files scheduled by the management software.

\subsection{Augmented Digital 3D Signage System}

The augmented system had to include the added functionality to handle and display $3 \mathrm{D}$ content. As before, the managing user interface had to be transparent to $2 \mathrm{D}$ and 3D media, in addition to static and motion media. In particular, the system had to be able to present contents produced for 3D display also on standard 2D displays. See Fig. 3. Furthermore, the system had to handle 3D media indifferent of the actual display installed because the managing software may control several display sites simultaneously. This means that a decentralized solution had to be implemented because it should be built on the existing digital signage system. Therefore, the augmented digital 3D signage system required a highly flexible video player that supports a multitude of formats and compression standards for multiview presentations on a variety of 2D and 3D displays running on different computer platforms.

\subsection{1. $3 D$ media player}

The number of publicly available software video players capable of decoding and playing multiview 3D video is still quite limited. None of the players fulfilled the requested features: stereoscopic and multiview autostereoscopic capability, open interface for added functionality, platform independence, and efficient computations.

Therefore, a highly portable, cross-platform 3D player was developed based on open source libraries [13]. It utilizes GPU-hardware accelerated graphics and has full control of de-multiplexing, decoding, scaling and rendering with a minimized overhead. The player further allows for easy upgrading to support more sophisticated encoding and rendering algorithms. The 3D player's modular approach to 3D video playback allows for different parts of the display surface to adhere to different display properties. This

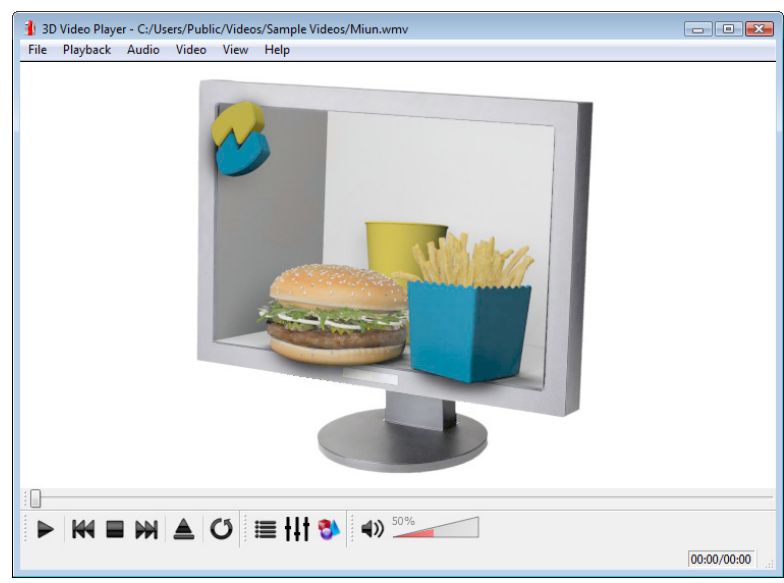

Fig. 4. Screenshot of 3D video player presenting a 3Ddisplay.

facilitates seamlessly combining $2 \mathrm{D}$ and $3 \mathrm{D}$ content as well as constructing 3D display walls using different 3D display types. The presented solution is a stand-alone $3 \mathrm{D}$ video player application built on top of FFmpeg, using libavformat for media container demultiplexing and libavcodec for video decoding. In order to increase modularity and flexibility, the 3D video player functionality was divided into two separate components:

- Video player - containing high-level video player functionality such as media container de-multiplexing, decoding, and synchronization.

- $3 D$ video filter - containing $3 \mathrm{D}$ video processing logic in the context of spatial multiplexing, RAM to video memory pixel data copying, GPU shader management etc.

Fig. 4 presents a screenshot of the $3 \mathrm{D}$ video player.

\section{CUSTOMER BEHAVIOR EFFECT}

The ultimate goal for most advertisement is to increase sales. The question is whether a digital 3D signage system will meet these expectations better than 2D signage. The prototype digital 3D signage system including autostereoscopic 3D displays was utilized to record and analyze the effect of customer exposed to 3D signage.

\subsection{Related works}

A market analysis has been carried out for autostereoscopic 3D displays for a number of market segments considering business model, needs, venue profiles, etc. [14]. The forecast for 3D displays in shopping malls worldwide is about 700 units in 2011, which will increase to about 4000 units in 2014, based on the number of venues, market penetration rate, and more. Today, content creators and installers are profiting from the 3D signage market, whereas it is forecasted that network operators, display makers and software developers will gain in the future. At present, the 
"wow-effect" is considered the most important factor for installing a digital 3D sign.

A series of studies has been carried out on the differences of using virtual technologies and physical world stimuli in conjoint analysis. Buyer's choices were compared when presented on 2D and autostereoscopic 3D displays with the choices when actual products were exhibited. I.e. 3D displays were rather used as tool for the analysis than evaluating the influence on customers by the technology itself. The study concluded that there is a higher degree of presence and involvement when 3D displays were utilized than 2D technology [15].

Interestingly, several earlier studies conclude that $3 \mathrm{D}$ presentations are not always preferred over 2D. The field tests carried out in [16] inferred that $2 \mathrm{D}$ viewing gave better quality because it contained fewer errors and required less viewing adjustments. Similar results were obtained when observers, not experienced with autostereoscopic 3D, assessed overall quality of 3D images affected by different compression algorithms: the least depth was preferred, despite many imperfections identified by an expert analysis [17]. As many as 70\% of the observers in the study of [18] favored $2 \mathrm{D}$ appearance over $3 \mathrm{D}$ due to depth rendering impairments; the 2D image had to be over five times smaller in order to give the same quality of experience. However, the experience of 3D was preferred over $2 \mathrm{D}$ by setting depth parameter such that depth variations were small.

\subsection{Customer behavior study}

The present study is somewhat different from the above mentioned investigations in the sense that the $3 \mathrm{D}$ presentation was used to catch a customer's attention rather than trying to maximize a quality of experience.

Collaboration was arranged between the research group, a digital signage provider and a fast food restaurant chain. One restaurant was chosen for a 3D video display experiment involving estimation of customer behavior impact. The restaurant in the study used a cash register storing sales data in a data base. Sales data could be obtained for the number of each product sold for each day, making it possible to follow up on sales over periods when a certain meal was presented in the 3D display. Our research hypothesis was that during days when a certain meal was highlighted by use of 3D, it would catch customer's interest and desire and thus sell at a higher rate than when presented in $2 \mathrm{D}$ mode. The purpose was to estimate the size of that effect.

\subsection{Method}

An existing 2D display was replaced by a 3D display in the restaurant. See Fig. 1. We studied the sales of three specific meals in the fast food restaurant by collecting cash register data. The numbers sold of these three meals on a specific day were compared to the total number of meals sold that day, giving the proportions of meals sold for each of the three meals, M1, M2, M3. The odds that a sale on that day was M1 was defined by the ratio of number of M1 sold to number of all other meals sold that day, and similarly for M2 and M3.

The meals M1, M2 and M3 were highlighted in the establishment by a special show alternating between the meals and between $3 \mathrm{D}$ and $2 \mathrm{D}$ video. The $2 \mathrm{D}$ video consisted of the exact same content as the central perspective view of the 3D content. The 3D Effect from these shows on sales of a meal was defined as ratio of the odds for an item sold to be of the specified meal, when displayed in 3D to the corresponding odds when it was displayed in 2D. The overall effect of displaying meals on the extra screen - whether 2D or 3D - here called Display Effect, was defined by the odds that a sold product was one of the three meals on a weekday when displayed in 2D or $3 \mathrm{D}$ compared to the corresponding odds during a period of three months immediately before the experiment.

We anticipated variation in number of customers across days and to eliminate effects of this variation we used odds and proportions as useful statistics rather that numbers of sold items. We also expected variation in customer profile across week days. To reduce systematic effects of this variation, for each of the three meals in the experiment we created sequences of shows that included one of the meals in $3 \mathrm{D}$ while the other two were shown in $2 \mathrm{D}$. Each meal was in this way highlighted by the use of 3D for a full day across the five week days. Saturdays and Sundays were excluded. However, random variation of customer profiles across days could not be eliminated, as well as all other random variation in customers and customer needs during the experiment.

To test the hypothesis that there was a 3D Effect and that there was a Display Effect due to the extra screen used, the non-parametric Kruskal-Wallis and Wilcoxon rank order tests were used. These tests were used in favour of the corresponding parametric tests since we were not positive that the measured odds ratios would have a normal distribution and that the number of weekdays, corresponding to number of observations in our study, was not large.

After conclusion of the experiment phase, we made interviews in the restaurant of four customers during lunch hours and four customers in late afternoon. An interview format was developed and responses analyzed using a model for consumer behavior impact based on Sheldon (1911), and including customer Exposure, Attention, Perception, Interest, Desire, and Action.

As described, experiment control was exercised in the study. However, we could not include randomization of customers in this real life setting, so the study of 3D impact on customer behavior is regarded as an observational study. 


\subsection{Results}

The experiment was designed for a three week periods, involving three different meals across five different week days, rendering 15 observations for each effect measure. We focused on the results from the meal which had the largest sales volume, according to sales history, here called M1, since it would have a smaller standard error in statistics used.

\subsubsection{Sales data}

The estimated 3D Effect on M1 was 0.984 which is close to one, meaning no effect (Kruskal-Wallis, $p$-value $=0.951$ ) and the estimated effect of the dish being presented in 2D or $3 \mathrm{D}$ does also not statistically significantly differ from zero (Wilcoxon, p-value $=0.083$ ) .

\subsubsection{Customer Interviews}

Eight customers were interviewed about their visit to the restaurant and general issues concerning their visit. The focus of the interviews was the customers' attention, perception and interest in the added 3D screen in the restaurant, the interest and desire it arouse and the impact it had on the choice of meal. The responses are analyzed and summarized as follows:

Attention: Three of the interviewed customers did not pay attention to the 3D screen. Reasons were that attention was on other signs or that they ordered other food than the standard meals. One of these customers noticed the sign but did not perceive the content. Her focus was on the regular information signs. The other two did not find it interesting due to the fact that they had decided what to eat before they entered.

Perception/Interest: Three of the interviewed noticed or even looked twice at the extra screen but it still did not have any impact on their choice. One of them ate only vegetarian food and the other two had decided what to eat already before arriving or entering at the restaurant.

Desire/ Action: Two of the customers interviewed looked attentively at the screen, one of them before, the other after ordering food. The one who looked before ordering said that he "liked what he saw" but still other information or habits made him make another choice in the end.

A summary of this analysis is that the customers had many different and personal reasons to choose what to eat at the restaurant. This can prevent some customers from paying attention or interest in signs, and prevent others from being affected by the message in a sign. Thus there is a variety of reasons for customers not to be affected by the extra signage provided.

\subsection{Discussion}

After following up sales data for 15 days generating numbers of the sold meals studied, comparing periods when the items were displayed in $3 \mathrm{D}$ and $2 \mathrm{D}$, we could see that the 3D Effect on sales was close to zero. By the results of customer interviews we may note that a decision to purchase a certain meal at the restaurant due to exposure of a 3D video show, requires that not only attention and perception of message is achieved but also that interest, desire and action occur..And there are many possibilities for this not to happen.

\subsubsection{Implications}

The results of the study may imply that $3 \mathrm{D}$ signage in settings like the one we studied is of limited value at least when it is in the form of an extra sign besides the regular information signs used. In research the study may be interpreted as a warning as to the very large difference there may be between results in controlled environments where perceptions could be registered on one hand, and on the other the complex reality of ordinary people's interest, desire and actions which are responses to so many other factors and individual differences, which emerge on higher or more complex levels of cognition.

\subsubsection{Limitations}

The study should have been carried out over a longer period of time, making it possible to gather a larger quantity of sales information. This would have decreased standard errors making it possible to estimate sizes of effects in a precise way using confidence intervals. However, the bottom line would almost certainly still be that the effect on the level of customer decisions would still be close to zero.

\section{CONCLUSIONS}

This paper presented aspects on the design and construction of a digital $3 \mathrm{D}$ signage system. It has considered issues for 3D content production, presentation, storage and distribution. Computer animated 3D content is more straight forward to produce than capturing of photographic data. The presentation of 3D content must be flexible in order to handle a multitude of de-multiplexing, decoding, scaling and rendering, yet with a minimized overhead. For storage and distribution, there are choices of format and encoding in order to support a variety of $2 \mathrm{D}$ and 3D displays. A high quality distribution is more important than to have a low latency transmission. We chose to implement a decentralized distribution solution as it requires less control signaling and less changes to the existing distribution system under consideration. A new 3D player was developed to render views from different formats.

A study on the customers' reaction and how sales are influenced by the new 3D signage technology was carried out. Analyzing experiment data, it was not possible during the short experiment period to verify that products exposed on the extra display increased sales. Neither could a difference be verified between 2D and 3D exposure. An 
interview study made it clear that customers were lost at different stages in this series of steps, among reasons were the placement of the sign. The influence of 3D signage must be further investigated with alternative placements of the digital sign in order to identify its influence with statistical significance.

\section{ACKNOWLEDGEMENTS}

This work has been supported by grants 42078 and 156702 of the EU European Regional Development Fund, Mellersta Norrland, Sweden, and by grants 44054 and 155148 of Länsstyrelsen Västernorrland, Sweden. The authors like to thank their collaborating partners NetCorner Europe AB and Nordic Fast Food AB.

\section{REFERENCES}

[1] L. Onural, et al., "An Assessment of 3DTV Technologies," Proc. NAB Broadcast Engineering Conference, Las Vegas, USA, April 2006, pp. 456-467.

[2] H. Urey, K.V. Chellappan, E. Erden, P. Surman, "State of the Art in Stereoscopic and Autostereoscopic Displays", Proc. IEEE, Vol. 99, no. 4, 540-555, 2011.

[3] "Digital Signage Advertising en Route to Becoming a MultiBillion Dollar Industry," Markwire web site, (NSR-report on Digital Signage Advertisement),

http://www.marketwire.com/press-release/Digital-SignageAdvertising-en-Route-to-Becoming-a-Multi-Billion-DollarIndustry-849687.htm, April 2008.

[4] "US Digital Signage Market to Grow by One Third in 2009", ABI Research web site, http://www.abiresearch.com/press/1385, March 2009

[5] “Global Market for Digital Signage, $2^{\text {nd }}$ ed.", NSR-report March 2010, NSR web site, http://www.nsr.com/Reports/MediaReports/GMDS2.html, 2010 .

[6] K. Ukai, and P.A. Howarth. "Visual fatigue caused by viewing stereoscopic motion images: Background, theories, and observations.” Displays, 29(2), 106-116, 2008.
[7] C. Ponce and R. Born, "Stereopsis," Current Biology vol. 18 no. 18 , pp. R845 - R850, September 2008.

[8] J. Kluger, "Stereo Production for 3D Cinema," 3D media workshop, Berlin, October 2009.

[9] I. Schiller, C. Beder, and R. Koch, "Calibration of a PMD Camera using a Planar calibration Pattern together with a Multi-camera Setup," Proc. XXI ISPRS Congress, Beijing, China, July 2008.

[10] O. Schreer, P. Kauff, and Th. Sikora (Editors), 3D Video Communication, Wiley, 2005.

[11] H. Urey et al, "State of the Art in Stereoscopic and Autostereoscopic Displays", Proceedings of the IEEE, vol. 99, no. 4, pp. 540-555, 2011.

[12] A. Smolic et al., "An overview of available and emerging 3D video formats and depth enhanced stereo as efficient generic solution," Proc. Picture Coding Symposium, May 2009, pp. $1-4$.

[13] R. Olsson, H. Andersson, and M. Sjöström, ”A modular cross-platform GPU-based approach for flexible 3D video playback," Proceedings of SPIE - Stereoscopic Displays and Applications XXII, 2011.

[14] D.H. Maunu et al, "2009 Autostereoscopic 3D Displays in Signage and Professional Applications," Insight Media web site, May 2009, http://www.insightmedia.info/yearlyreports.php\#2009as3d.

[15] Alma Berneburg, "Presence in a Three-Dimensional Test Environment: Benefit or Threat to Market Research?", Journal of Virtual Reality and Broadcasting, vol. 5, no. 1, March 2008.

[16] S. Jumisko-Pyykkö and T. Utriainen, "Results of the usercentred quality evaluation experiments," Technical report D4.4, MOBILE3DTV (EU-FP7 Project No. 216503), November 2009.

[17] R. Olsson and M. Sjöström, "Multiview image coding scheme transformations: artifact characteristics and effects on perceived 3D quality," in Proc. SPIE: Conference on Stereoscopic Displays and Applications XXI, San Jose, CA, JAN 18-20, 2010.

[18] M. Barkowsky, R. Cousseau, and P. Le Callet, "Influence of depth rendering on the quality of experience for an autostereoscopic display“, Proc. International Workshop on Quality of Multimedia Experience, San Diego, CA, USA, 2931 July 2009, pp. 192-197. 